\title{
Psoriasis: Systemische Entzündungsreaktionen bremsen
}

- Vor acht Jahren wurde Etanercept (Enbrel $^{\circledR}$ ) zur Behandlung der Psoriasis-Arthritis, vor fünf Jahren zur Behandlung der Psoriasis zugelassen. Zunehmend hat sich die Erkenntnis durchgesetzt, dass die Psoriasis nicht auf die Haut beschränkt bleibt, sondern eine Systemerkrankung ist. Die Versorgung von Psoriasispatienten hat sich deutlich verbessert, es gibt heute mehr als 6o Psoriasisschwerpunktpraxen in Deutschland. Mittlerweile liegen nach Ausführung von Wolfram Sterry, Berlin, umfangreiche Erfahrungen zur Anwendung von Etanercept bei der Psoriasis vor. So zeigte die Studie „Clinical Randomized Year-long STudy assessing the safety and efficacy of EnbreLin psoriasis (CRYSTEL)“, dass die Erkrankung sowohl intermittierend, als auch kontinuierlich mit ähnlichem Erfolg behandelt werden kann. „Beide Methoden funktionieren zwar, doch Patienten sind bei einer Dauertherapie mit Etanercept zufriedener", so Sterry.

Grundsätzlich sind zur Bekämpfung der Hautsymptome höhere Dosen nötig als zur Therapie der Psoriasisarthritis: Dies zeigte die Studie „Psoriasis Randomized Etanercept STudy in Subjects with Psoria- tic Arthritis (PRESTA)“, in der die doppelte Dosierung von Etanercept in den ersten 12 Therapiewochen einen signifikanten Einfluss auf die Hautbesserung aufwies, wogegen sich Gelenkparameter durch eine doppelte Dosis nicht weiter verbesserten.

Wie Gerd Horneff, St. Augustin, ausführte, ist Etanercept seit 2009 als einziges Biologic auch zur Behandlung der juvenilen PlaquePsoriasis zugelassen.54\% der Kinder erreichen eine vollständige Kontrolle binnen 12 Wochen Ein großer Vorteil der Therapie mit Etanercept bei Kindern ist, dass durch den

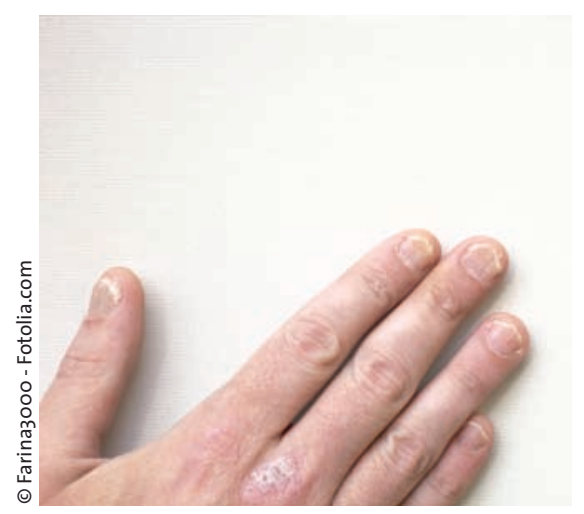

Verzicht auf Steroide das Längenwachstum bei zuvor kleinwüchsigen Kindern verbessert werden kann.

Da Etanercept als erstes Biologikum vor 10 Jahren zur Behandlung der rheumatoiden Arthritis und der polyartikulären juvenilen idioapthischen Arthritis zugelassen wurde, kann mittlerweile auf eine Erfahrung von über zwei Millionen Patientenjahren zurückgeblickt werden. Hier zeigte sich, dass die Inzidenz von Infekten nur minimal erhöht ist. Auch die langfristige Therapie mit Etanercept führt zu keinem Anstieg von Lymphomen oder anderen Tumoren. ka

Quelle: Jubiläums-Pressekonferenz Enbrel ${ }^{\circledR}$ „Zehn Jahre Medizinische Avantgarde Fortschritt für Ärzte und Patienten". Berlin, 8.7.2010 (Veranstalter: Pfizer).

Psoriasis: nicht nur psychisch für viele Patienten eine schwere Belastung ...

\section{IL-12/23-Blockade zeigt langfristige Wirkung}

- Psoriatiker profitieren von einer wirksamen Therapie in vielfacher Hinsicht, denn eine Besserung des Hautzustands ist für viele Patienten der Schlüssel zu einer besseren Lebensqualität. Dies schlägt sich auch in der Sexualität nieder. Studien haben gezeigt, dass sich viele Patienten mit Psoriasis in ihrer Sexualität beeinträchtigt fühlen. In einer Phase-III-Studie fiel der Anteil dieser Patienten von 22,4 auf 2,7\% nach zwölf Wochen Therapie mit Ustekinumab (Stelara ${ }^{\circledR}$ ). Ustekinumab ist das erste Biologic, das primär für die Therapie der Psoriasis entwickelt wurde, berichtete Prof. Dr. Kristian Reich, Hamburg. Und es ist der erste Wirkstoff, der die Interleukine (IL) 12 und 23 blockiert. Damit wird der Krankheitsprozess an einer Stelle unterbrochen, die sehr früh im entzündlichen Geschehen steht. Das neue Wirkprinzip scheint sogar besser zu wirken als die reine TNF- $\alpha$-Blockade. Das zeigte die ACCEPT-Studie, in der erstmals zwei Biologika miteinander verglichen wurden: 903 Patienten mit mittelschwerer bis schwerer Plaquepsoriasis hatten entweder Ustekinumab (45 oder $90 \mathrm{mg}$ in Woche o und 4) oder Etanercept (50 mg, zweimal pro Woche) erhalten. Unter Ustekinumab besserte sich der Hautzustand bei signifikant mehr Patienten als unter Etanercept. Die PASI-75Ansprechraten lagen unter 90 mg Ustekinumab bei $74 \%$, unter $45 \mathrm{mg}$ Ustekinumab bei $68 \%$ und unter Etanercept bei $57 \%$. Hinsichtlich der PASI-90-Ansprechrate war der Anti-IL-12/23-Antikörper ebenfalls signifikant überlegen.

Die Lebensqualität steigt vor allem dann, wenn Psoriatiker über längere Zeit schubfrei bleiben. Hier zeichnet sich ein Paradigmenwechsel in der Therapie ab, erklär- te Dr. Marc Alexander Radtke, Hamburg. Die früher bevorzugte intermittierende Therapie, die nach einem Schub wieder abgesetzt wurde, wird mehr und mehr von einer Langzeittherapie abgelöst. Mit Biologika ist eine Langzeittherapie gut möglich, so Radtke, denn die Nebenwirkungsrate ist relativ niedrig. Dabei bleibt die Wirksamkeit von Ustekinumab über mindestens drei Jahre erhalten. Das ist aus den Langzeitdaten der PHOENIX-1- und -2-Studien abzulesen, berichtete Reich. Er betonte, dass es über drei Jahre zu keiner Zunahme an unerwünschten Ereignissen gekommen ist und auch schwere Infektionen nicht zugenommen haben. jn

Pressekonferenz „1 Jahr Stelara ${ }^{\circledR}$ : Von der reaktiven zur proaktiven Therapie. München, 10.2.2010 (Veranstalter: Janssen-Cilag) 